\title{
Dual-Transmitter Inductive Power Transfer System with Improved Misalignment Tolerance and High Compatibility with Different-Sized Receivers
}

\author{
Guodong Zhu, Dawei Gao ${ }^{1}$ \\ ${ }^{1}$ (Corresponding author) State Key Laboratory of Automotive Safety and Energy, School of Vehicle and Mobility, Tsinghua \\ University, Beijing, China.Email: dwgao@mail.tsinghua.edu.cn
}

\begin{abstract}
Summary
A dual-transmitter inductive power transfer system featuring two concentric transmitter coils is proposed for low-power systems where compatibility with different-sized receivers and good misalignment tolerance are required. By incorporating two decoupled TXs into one single unit, the proposed scheme achieves better power and efficiency performance under a lower bill-of-materials cost compared to using two individual TXs, especially when the RX coil is small and the coil misalignment is large. Both TXs are energized using independent inverters and the inverter output voltages are regulated in accordance with the coupling condition to maximize the system efficiency. Superiority of the dual-TX system over single-TX systems is proved by experimental results.
\end{abstract}

Keywords: efficiency, inductive charger, optimization, wireless charging

\section{Introduction}

Inductive power transfer (IPT) is gaining increasing attention in applications ranging from portable devices to electric vehicles. However, one shortcoming of a typical IPT system is the need for accurate positioning. Although most IPT systems can withstand a certain degree of positioning error, degradation of efficiency and output power due to coil misalignment is unavoidable when the transmitter (TX) and receiver (RX) coils have similar dimensions [1]. One method for improving the tolerance to coil misalignment is to increase the TX coil size. However, the disadvantages are higher leakage magnetic field strength and lower system efficiency because a large proportion of the magnetic flux generated by the TX coil is not coupled to the RX coil. Another method is to use selectively energized multiple TX coils [2], and furthermore, to optimize the energizing strategy [3-4]. The TX coils that are relatively strongly coupled to the RX coil are energized while those weakly coupled to the RX coils are in stand-by mode. It is proved that the system efficiency can be effectively improved by optimizing the TX coil current ratio [3]. There are some other solutions that improve the stability of output power via compensation circuit design, such as the modified LCC compensation topology proposed in [5] and the hybrid compensation circuit structure reported in [6], however, efficiency degradation under coil misalignment is still inevitable in these schemes.

Apart from improving the tolerance to coil misalignment, another important concern for IPT systems is to properly design the TX side so that it can efficiently transfer power to different-sized RXs. Such a demand becomes more urgent when various types of electronic devices are wirelessly powered. Compared to using multiple individual TXs, using a single TX pad that is compatible with different RXs greatly reduces the total 
bill-of-materials cost. However, a single TX coil usually cannot transmit power to all RXs at a high efficiency because the geometrical dimensions vary greatly from one RX to another. In this work, a dual-TX IPT system featuring two concentric and different-sized TX coils is proposed. The TXs are magnetically decoupled from each other and energized by independent inverters to provide two power transfer channels, which improves the stability of efficiency and output power against coil misalignment, especially when the RX coil is small. The bill-of-materials cost is lower than using two individual TXs (because only one ferrite plate is required). The performance improvement is achieved by simultaneously utilizing both power transfer channels (instead of using one only and leaving the other in idle state) and properly allocating the power that each channel carries. The maximum-efficiency condition is analyzed based on a simplified circuit model, and a possible maximum efficiency tracking method based on perturb and observe $(\mathrm{P} \& \mathrm{O})$ is discussed. Superiority of the proposed design is verified via experimental results.

\section{Efficiency Analysis of Dual-TX IPT System}

\subsection{Minimizing Conduction Loss for Series-Compensated Dual-TX IPT System}

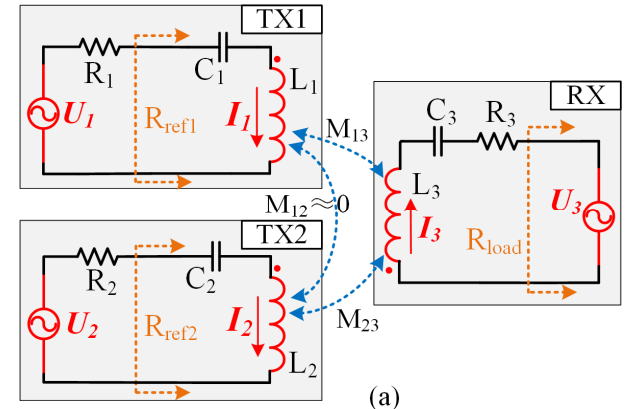

(a)

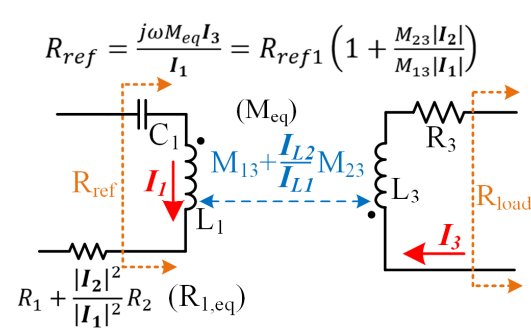

(b)

Fig. 1. Simplified schematic of series-compensated dual-TX IPT system. (a) Original system. (b) Single-TX equivalent system.

A simplified schematic of a series-compensated dual-TX IPT system is presented in Fig. 1. Due to the bandpass nature of the compensation networks, only the fundamental currents and voltages are considered. With proper adaptations, the conclusions derived here can be generalized to IPT systems with other compensation topologies. $\boldsymbol{U}_{\mathbf{1}}$ and $\boldsymbol{U}_{2}$ are the inverter output voltages, $\boldsymbol{U}_{\mathbf{3}}$ is the rectifier input voltage, $\boldsymbol{I}_{\mathbf{1}}$ and $\boldsymbol{I}_{\mathbf{2}}$ are the TX coil currents, and $\boldsymbol{I}_{3}$ is the RX coil current. $L_{1}, L_{2}$ and $L_{3}$ are the self-inductances of the TX coils and the RX coil, respectively. The mutual inductances among the three coils are denoted as $M_{12}, M_{13}$ and $M_{23}$. $C_{1}, C_{2}$ and $C_{3}$ are the compensation capacitances. $R_{1}, R_{2}$ and $R_{3}$ are the equivalent series resistances (ESRs) of the three loops. The power losses in the inverters and the rectifier are temporarily neglected. For simplicity, all ESRs are omitted except in efficiency analysis. $R_{\text {load }}$ is the equivalent AC load resistance observed at the rectifier input port. $R_{\text {ref } 1}$ and $R_{\text {ref } 2}$ are the reflected resistances on the TX side.

To simplify the analysis and control procedures, the TX coils are magnetically decoupled. When the circuits are fully resonant, i.e., $L_{1} C_{1}=L_{2} C_{2}=L_{3} C_{3}=1 / \omega^{2}$, where $\omega$ is the angular operating frequency, the reflected resistances are calculated as

$$
\begin{gathered}
R_{\text {ref } 1}=\frac{j \omega M_{13} I_{3}}{I_{1}}=\frac{j \omega M_{13}\left(-j \omega M_{13} I_{1}-j \omega M_{23} I_{2}\right)}{I_{1} R_{\text {load }}}=\frac{\omega^{2} M_{13}^{2}}{R_{\text {load }}}+\frac{\omega^{2} M_{13} M_{23}}{R_{\text {load }}} \frac{\boldsymbol{I}_{2}}{\boldsymbol{I}_{1}}, \\
R_{\text {ref } 2}=\frac{j \omega M_{23} \boldsymbol{I}_{3}}{\boldsymbol{I}_{\mathbf{2}}}=\frac{\omega^{2} M_{23}^{2}}{R_{\text {load }}}+\frac{\omega^{2} M_{13} M_{23}}{R_{\text {load }}} \frac{\boldsymbol{I}_{\mathbf{1}}}{\boldsymbol{I}_{2}} .
\end{gathered}
$$

When $\left|\boldsymbol{I}_{\mathbf{1}}\right|$ and $\left|\boldsymbol{I}_{2}\right|$ are given, $\left|\boldsymbol{U}_{\mathbf{3}}\right|$ is maximized under the condition $\angle \boldsymbol{I}_{\mathbf{1}}=\angle \boldsymbol{I}_{\mathbf{2}}$, hence a necessary condition for minimizing the conduction loss is

$$
\angle I_{1}=\angle I_{2}
$$


This condition is applied in the following parts of this paper. The output power $\left(P_{\text {out }}\right)$ is calculated as

$$
P_{\text {out }}=\frac{\left|\boldsymbol{U}_{3}\right|^{2}}{2 R_{\text {load }}}=\frac{\omega^{2}\left(M_{13} I_{1}+M_{23} \boldsymbol{I}_{2}\right)^{2}}{2 R_{\text {load }}}=\frac{1}{2}\left|\boldsymbol{I}_{\mathbf{1}}\right|^{2} \frac{\omega^{2}\left(M_{13}+M_{23} \mid \frac{\left|I_{2}\right|}{\left|I_{1}\right|}\right)^{2}}{R_{\text {load }}} .
$$

Meanwhile, the TX-side conduction loss $\left(P_{\text {cond,TX }}\right)$ is calculated as

$$
P_{\text {cond }, T X}=\frac{1}{2}\left|\boldsymbol{I}_{1}\right|^{2} R_{1}\left(1+\frac{\left|\boldsymbol{I}_{2}\right|^{2} R_{2}}{\left|\boldsymbol{I}_{1}\right|^{2} R_{1}}\right) .
$$

Therefore, the condition for minimizing the $P_{\text {cond,TX }}$-to- $P_{\text {out }}$ ratio is

$$
\frac{\left|I_{2}\right|}{\left|I_{1}\right|}=\frac{R_{1} M_{23}}{R_{2} M_{13}} .
$$

A similar conclusion is derived in [3]. Replacing the $\boldsymbol{I}_{\mathbf{2}}$-to- $\boldsymbol{I}_{\mathbf{1}}$ ratio in (1-a) and (1-b) with the right-hand side of (5) yields an equivalent condition:

$$
\frac{R_{r e f 2}}{R_{2}}=\frac{R_{r e f 1}}{R_{1}} .
$$

The dual-TX system in Fig. 1 (a) can be converted into a single-TX equivalent system as shown in Fig. 1 (b), in which $L_{1}$ is the only TX coil. The equivalent mutual inductance is derived as

$$
M_{e q}=M_{13}+\frac{I_{2}}{I_{1}} M_{23} .
$$

The TX-side ESR and the reflected resistance are derived as

$$
\begin{gathered}
R_{1, e q}=\left(1+\frac{\left|\boldsymbol{I}_{2}\right|^{2} R_{2}}{\left|\boldsymbol{I}_{1}\right|^{2} R_{1}}\right) R_{1}, \\
R_{\text {ref }}=\frac{j \omega M_{e q} \boldsymbol{I}_{3}}{\boldsymbol{I}_{\mathbf{1}}}=R_{\text {ref } 1}\left(1+\frac{M_{23}\left|\boldsymbol{I}_{2}\right|}{M_{13}\left|\boldsymbol{I}_{1}\right|}\right) .
\end{gathered}
$$

Invariance of $P_{\text {out }}$ and $P_{\text {cond,TX }}$ after the conversion can be easily verified. The overall coil conduction loss in the single-TX equivalent system is calculated as

$$
P_{\text {cond }}=\frac{1}{2}\left[\left|\boldsymbol{I}_{\mathbf{1}}\right|^{2} R_{1, e q}+\left(\frac{\omega M_{\text {eq }}\left|\mathbf{I}_{\mathbf{1}}\right|}{R_{\text {load }}}\right)^{2} R_{3}\right] .
$$

Equation (5) is applied in the following analysis. Under a given $P_{\text {out }}$, i.e., $\left(\omega M_{\text {eq }}\left|\boldsymbol{I}_{\mathbf{1}}\right|\right)^{2} /\left(2 R_{\text {load }}\right)$ is fixed, $P_{\text {cond }}$ is minimized under the condition

$$
R_{\text {load }}=R_{\text {load }, \text { opt }}=\omega M_{e q} \sqrt{\frac{R_{3}}{R_{1, e q}}}=\omega \sqrt{\left(\frac{M_{13}^{2}}{R_{1}}+\frac{M_{23}^{2}}{R_{2}}\right) R_{3}} .
$$

Based on (10), the following two equivalent conditions are derived:

$$
\begin{gathered}
\frac{R_{\text {load }}}{R_{3}}=\frac{R_{\text {ref }}}{R_{1, \text { eq }}}, \\
\frac{\left|\boldsymbol{I}_{3}\right|}{\left|\boldsymbol{I}_{\mathbf{1}}\right|}=\sqrt{\frac{R_{1}}{R_{3}}} \sqrt{1+\frac{M_{23}^{2} R_{1}}{M_{13}^{2} R_{2}} .}
\end{gathered}
$$

Conditions (6) and (11-a) are independent of each other. Even if $R_{\text {load }}$ is not matched to $R_{\text {load,opt }}$, the TXside conduction loss can still be minimized by satisfying (6).

\subsection{Minimizing Conduction Loss for LCC-Compensated Dual-TX IPT System}

A simplified schematic of an LCC-compensated dual-TX IPT system is shown in Fig. 2. $L_{f 1}, L_{f 2}, C_{f 1}$ and $C_{f 2}$ are the extra components that are required in the LCC compensation networks. Under the condition of full 
resonance, i.e., $L_{f 1} C_{f 1}=L_{f 2} C_{f 2}=\left(L_{1}-L_{f 1}\right) C_{1}=\left(L_{2}-L_{f 2}\right) C_{2}=1 / \omega^{2}$, the input impedances of the TX-side compensation networks are purely resistive and denoted as $R_{i n 1}$ and $R_{i n 2}$, respectively. The relation between $R_{i n 1 / 2}$ and $R_{r e f 1 / 2}$ can be derived from the basic properties of the LCC compensation topology as

$$
R_{i n 1 / 2}=\frac{\omega^{2} L_{f 1 / 2}^{2}}{R_{r e f 1 / 2}}
$$

It is noteworthy that in Fig. 2, the impact of inverter conduction loss is modelled as part of $R_{i n v 1}$ or $R_{i n v 2}$ instead of $R_{1}$ or $R_{2}$. As a result, conditions (6) and (11-a) cannot be directly applied due to these extra loss terms. These conditions do serve as a good reference, though. In this work, LCC compensation is adopted on the TX side. A more practical method for maximum efficiency tracking that avoids the trouble of mathematically deriving the maximum-efficiency conditions and eliminates the impact of stray factors, e.g., instability of ESRs, is to regulate the inverter output voltages using heuristic algorithms, e.g., the wellestablished $\mathrm{P} \& \mathrm{O}$ method.

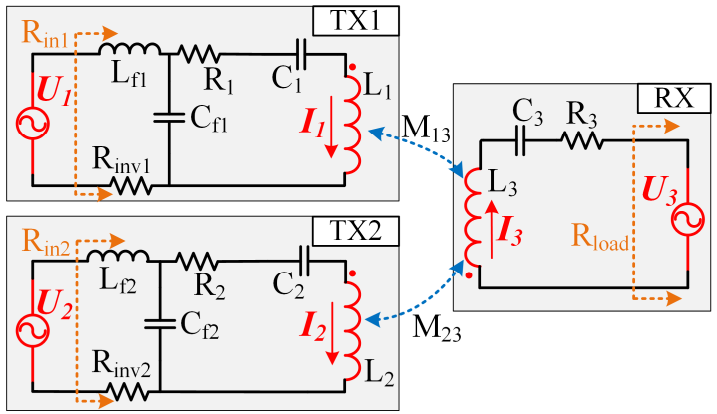

Fig. 2. Dual-TX IPT system with LCC-compensated TXs.

In this work, the TX side is designed for compatibility with different-sized RX pads, hence it is possible that $L_{1}$ and $L_{2}$ are not constant when different RX pads are paired with the TXs. The compensation capacitances $C_{1}$ and $C_{2}$ can be chosen to satisfy the full-resonance condition when $L_{1}$ and $L_{2}$ are at their medium values (measured when a medium-sized RX is paired with the TXs).

\section{System Parameters and Control Strategy}

\subsection{Coil Geometry}

The proposed TX-side design is aimed at low-power charging applications where compatibility with differentsized receivers is required. The TX coils are concentric and located above the same ferrite core, as is illustrated in Fig. 3. No aluminum plate is deployed on the TX side.
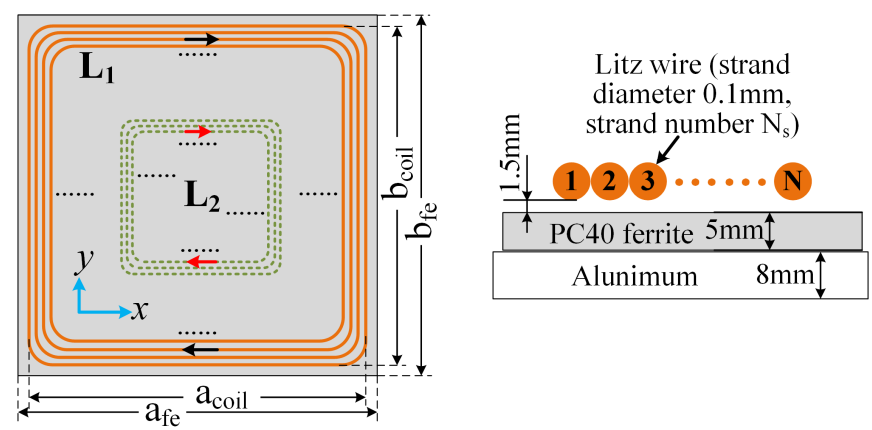

Fig. 3. Geometric parameters of the TX-side coil assembly. 
Three different-sized receiver pads are adopted in this work. The overall structure is identical with that shown in Fig. 3. The RX pads are designated as RX1, RX2 and RX3, respectively, with RX1 being the largest and RX3 being the smallest. An aluminum shielding plate is attached to the RXs. The height of air gap between the ferrite core and the aluminum plate is zero. For RX1, the aluminum plate dimensions are $350 \mathrm{~mm} \times 300 \mathrm{~mm} \times 8 \mathrm{~mm}$ and the long side is parallel with the $\mathrm{x}$-axis. For RX2 and RX3, the aluminum plate dimensions are $300 \mathrm{~mm} \times 140 \mathrm{~mm} \times 8 \mathrm{~mm}$ and the long side is parallel with the $y$-axis.

The geometric parameters of the coil assemblies are presented in Table $1 . h_{\text {coil }}$ denotes the vertical distance between the TX and RX coils. The coils are made of Litz wires with different strand numbers $\left(N_{S}\right)$ and the strand diameter is fixed at $0.1 \mathrm{~mm}$. The self-inductances and the ESRs are stable under different coil misalignment values (along the direction in which the long side of the aluminium plate is located) whereas the mutual inductances change drastically with misalignment. The subscripts " 0 " and " 1 " mean that the mutual inductance data are measured at the perfectly-aligned and maximum-misalignment positions, respectively. The other quantities are measured at the perfectly-aligned position. The maximum misalignment for RX1, RX2 and $\mathrm{RX} 3$ is $30 \mathrm{~mm}, 40 \mathrm{~mm}$ and $50 \mathrm{~mm}$, respectively.

The inductances and ESRs are measured using an LCR meter (HIOKI IM3536) at $85 \mathrm{kHz}$ and shown in Table 2. The ESRs of $C_{1}, C_{2}$ and $C_{3}$ are included in $R_{1}, R_{2}$ and $R_{3}$, respectively. $R_{i n v 1 / 2}$ contains two parts: the on-state resistance of the MOSFETs (obtained from the device datasheet) and the ESR of $L_{f 1 / 2}$ (experimentally measured).

Table1: Geometric parameters of the coils

\begin{tabular}{lccccccc}
\hline & $a_{f e}(\mathrm{~mm})$ & $b_{f e}(\mathrm{~mm})$ & $a_{\text {coil }}(\mathrm{mm})$ & $b_{\text {coil }}(\mathrm{mm})$ & $\mathrm{N}$ & $h_{\text {coil }}(\mathrm{mm})$ & $N_{s}$ \\
\hline TX1 & 210 & 210 & 186 & 186 & 11 & -- & 250 \\
TX2 & & & 75 & 75 & 13 & -- & 100 \\
RX1 & 210 & 210 & 186 & 186 & 11 & 63 & 250 \\
RX2 & 115 & 120 & 108 & 112 & 12 & 39 & 120 \\
RX3 & 100 & 110 & 68 & 74 & 12 & 32 & 100 \\
\hline
\end{tabular}

Table2: Circuit parameters of the dual-TX IPT system when different RXs are used

\begin{tabular}{ccccccccccccc}
\hline & $L_{1}$ & $L_{2}$ & $L_{3}$ & $M_{13,0}$ & $M_{23,0}$ & $M_{13,1}$ & $M_{23,1}$ & $R_{1}$ & $R_{2}$ & $R_{3}$ & $R_{\text {inv1 }}$ & $R_{\text {inv2 }}$ \\
\hline RX1 & 61.3 & 24.8 & 57.2 & 15.9 & 2.3 & 10.6 & 1.0 & 97.3 & 127.4 & 136.5 & & \\
RX2 & 56.4 & 25 & 33.9 & 8.2 & 5.4 & 7.4 & 2.2 & 128.5 & 128.8 & 155.2 & 59.8 & 61.6 \\
RX3 & 53.7 & 25.4 & 19.5 & 3.2 & 5.2 & 3.6 & 2.9 & 137 & 130.6 & 97.8 & & \\
\hline
\end{tabular}

Note: units of the inductances and ESRs are $\mu \mathrm{H}$ and $\mathrm{m} \Omega$, respectively.

\subsection{Maximum Efficiency Tracking Control}

Considering the simplifications made in the mathematical model and the other stray factors, e.g., parameter variation, the following closed-loop control strategy can be adopted to maximize the system efficiency. The RX-side controller maintains $P_{\text {out }}$ at the target value by regulating $R_{\text {load }}$ using power converters, e.g., $\mathrm{DC} / \mathrm{DC}$ converters [4], and the control algorithm is simple.

The main objective of the TX-side controller is to maximize the system efficiency by searching for the optimal $R_{i n 1}$ and $R_{i n 2}$. The ratio $R_{i n 1} / R_{i n 2}$ is related to the allocation of input power between the TXs, which in turn affects the TX-side power loss. In the practical IPT system, the control variables are the inverter conduction angles $\left(\theta_{i n v 1}\right.$ and $\left.\theta_{i n v 2}\right)$. One can easily infer from (1-a), (1-b) and (12) that $R_{i n 1}$ increases monotonically with $\left|\boldsymbol{U}_{1}\right|$ (or $\theta_{i n v 1}$ ) and the same relation exists between $R_{i n 2}$ and $\left|\boldsymbol{U}_{2}\right|$ (or $\theta_{i n v 2}$ ). The block diagram of a viable TX-side control strategy (based on P\&O) is shown in Fig. 4. During each control cycle, an increment is added to $R_{i n 1}$ or $R_{i n 2}$ and the impact on the input power $\left(P_{i n}\right)$ is observed. If the resultant $P_{i n}$ is lower than the value measured in the previous step, then the increment should be continued, otherwise the increment should be reversed. $R_{i n 1}$ and $R_{i n 2}$ can be regulated at different frequencies by using a counter variable (n). 
There are 3 control variables in the system, i.e., $\left|\boldsymbol{U}_{\mathbf{1}}\right|,\left|\boldsymbol{U}_{\mathbf{2}}\right|$ and $R_{\text {load. After }}\left|\boldsymbol{U}_{\mathbf{1}}\right|$ and $\left|\boldsymbol{U}_{\mathbf{2}}\right|$ are chosen, $R_{\text {load }}$ is subject to the $P_{\text {out }}$ constraint. Therefore, the RX-side operating status is uniquely determined during maximum efficiency tracking.

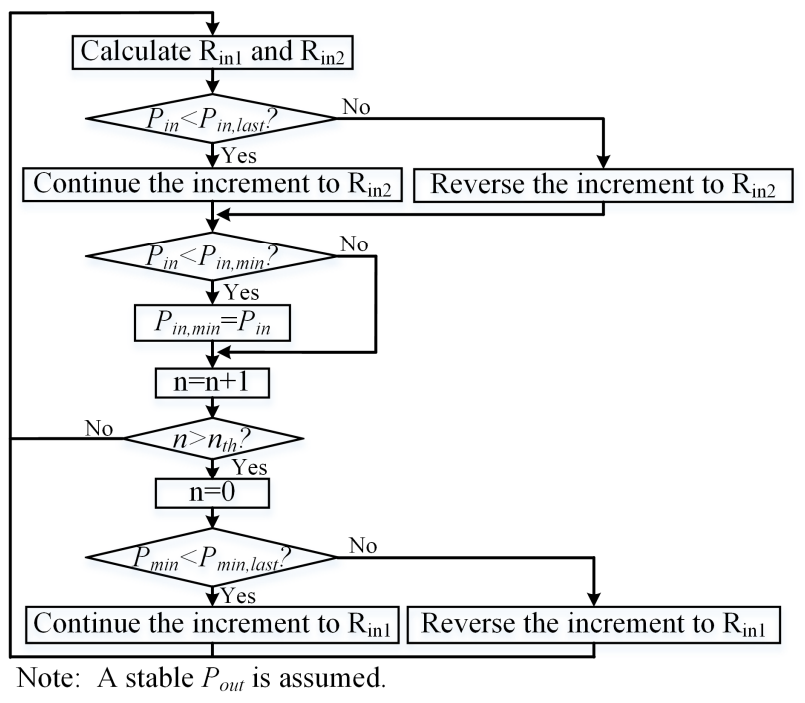

Fig. 4. P\&O-based TX-side closed-loop control strategy.

In this paper, however, open-loop control is adopted in the experiments because the focus of this work is to reveal the intrinsic properties of the proposed dual-TX scheme. Besides, the DC load resistance is fixed at $10 \Omega$, hence $R_{\text {load }}$ regulation is not utilized during maximum efficiency tracking.

\subsection{Compensation Circuit Design}

The series compensation topology is adopted on the RX side. The operating frequency is fixed at $85 \mathrm{kHz}$. Under the condition $\omega^{2} L_{3,0} C_{3}=1(\omega=2 \pi \times 85000 \mathrm{rad} / \mathrm{s})$, the value of $C_{3}$ can be easily calculated to be $61.2 \mathrm{nF}$, 103.2nF and $179.5 \mathrm{nF}$ for the three RXs, respectively. On the TX side, the DC-link voltage is varied between $48 \mathrm{~V}$ and $80 \mathrm{~V}$. The variable DC-link voltage reduces the burden on the inverters, i.e., $\theta_{\text {inv } 1}$ or $\theta_{\text {inv } 2}$ can be maintained at 180 degrees over a wider range of operating points to avoid hard-switching of the inverters.

The maximum output power for the three RXs are 400W, 220W and 60W, respectively. The DC load resistance is fixed at $10 \Omega$. The parameters to be determined are $L_{f 1}$ and $L_{f 2}$. The other compensation parameters, i.e., $C_{1}, C_{2}, C_{f 1}$ and $C_{f 1}$, are solved from the resonance conditions. $L_{f 1}$ and $L_{f 2}$ are manually optimized using the LTspice software. They should be chosen in such a way that the maximum output power can be reached at the maximum-misalignment position when the DC-link voltage reaches the upper boundary. The purpose is to guarantee that the output power is always achievable and the inverter conduction angles can be as large as possible. The final choice is $L_{f 1}=13 \mu H$ and $L_{f 2}=15 \mu H$. The other parameters are as follows: $C_{1}=$ 80.6nF $, C_{2}=350 n F, C_{f 1}=269 n F, C_{f 2}=233 n F$. The conditions $L_{1}=56.4 \mu H$ and $L_{2}=25 \mu H$ (measured when RX2 is paired with the TXs, see Table 2) are utilized in calculating $C_{1}$ and $C_{2}$.

\section{Experimental Results}

The IPT prototypes used in the experiments are configured as shown in Fig. 5. The MOSFET used in the fullbridge inverters and the full-bridge rectifier is DMTH10H025LK3 from Diodes Inc. The rectifier operates in passive rectification mode. The phase difference between the inverter output voltages is fixed at zero while the inverter conduction angles together with the DC-link voltage are manually regulated to realize the target output power following a simple rule: only when the target output power cannot be achieved is the DC-link voltage 
allowed to be increased. The purpose is to minimize the possibility of hard-switching for the inverters. The power and efficiency data are acquired using a power analyser (HIOKI PW6001). The TX coils are decoupled by adding reverse turns to TX1, as is shown in Fig. 5 (b).

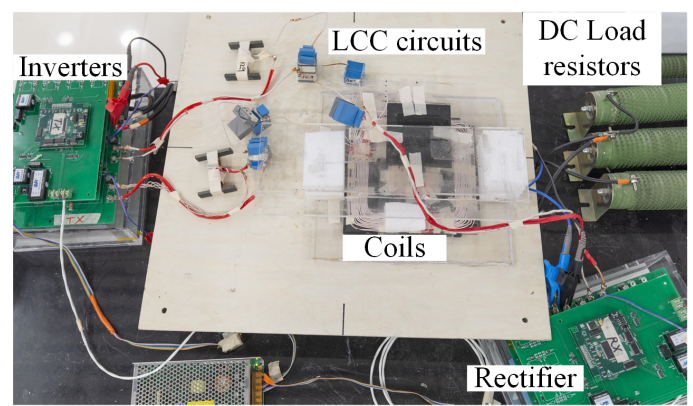

(a)

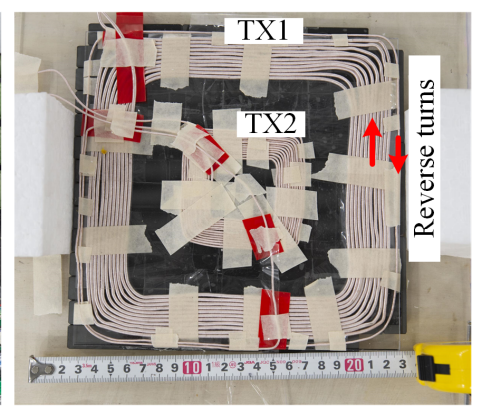

(b)

Fig. 5. IPT prototype. (a) Whole system (the aluminum plate is removed to show the coil structure more clearly). (b) TXside coil assembly.

An example is given in Fig. 6 to demonstrate the relation between inverter conduction angle (or equivalently, $R_{i n}$ ) and efficiency. $\theta_{i n v 1}$ is fixed at 180 degrees while $\theta_{i n v 2}$ together with the DC-link voltage is regulated to maintain $P_{\text {out }}$ at $220 \mathrm{~W}$. RX2 is the receiver and the coil misalignment is fixed at 0 . The result shows that the exactly optimal $\left|\boldsymbol{I}_{2}\right|-$ to $-\left|\boldsymbol{I}_{\mathbf{1}}\right|$ ratio (calculated to be approximately 0.86 ) deviates from the result predicted using (5), which is approximately 0.66 . Still, the condition in (5) serves as a good initial point for maximum efficiency tracking.

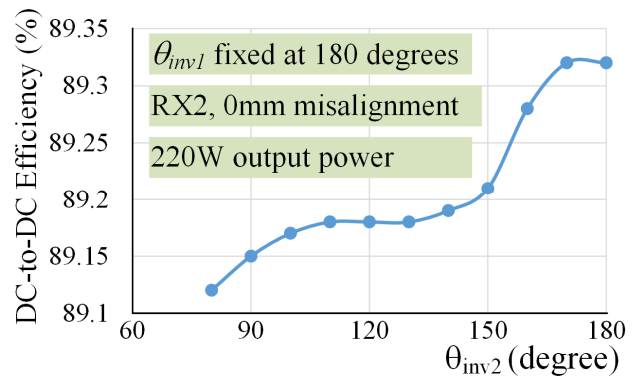

Fig. 6. Relation between efficiency and $\theta_{i n v 2}$ at a selected operating point.

Comparisons between the dual-TX system and the single-TX systems are made and the results are presented in Fig. 7. In the dual-TX system, the conduction angle of one inverter is fixed at 180 degrees while that of the other is regulated together with the DC-link voltage to search for the maximum-efficiency point. The DC-link voltage is between $48 \mathrm{~V}$ to $80 \mathrm{~V}$. In the single-TX systems, one TX coil is energized while the other is opencircuited. Overall, the dual-TX system achieves higher efficiencies than its single-TX counterparts. Except when one of the TXs is so weakly coupled to the RX that its contribution to the total loss outweighs its contribution to the output power, such as the "RX1 DT" case in Fig. 7 (a) (the efficiency difference is very small, though), the dual-TX system is superior in terms of the maximum achievable efficiency because there are two power transfer channels and the power through one channel can be easily decreased (by changing the $R_{i n 1} / R_{\text {in } 2}$ ratio) when assigning more power to the other channel results in a higher system efficiency. Besides, the dual-TX system achieves higher output powers at large-misalignment positions, especially when the RX is small and its coupling with TX2 decreases fast with misalignment. When RX1 is paired with the TXs, the target output power can be realized in both single-TX and dual-TX systems at all misalignment values. When RX2 or RX3 is used, the single-TX system fails to deliver the target output power at all misalignment values and the efficiency is lower. 

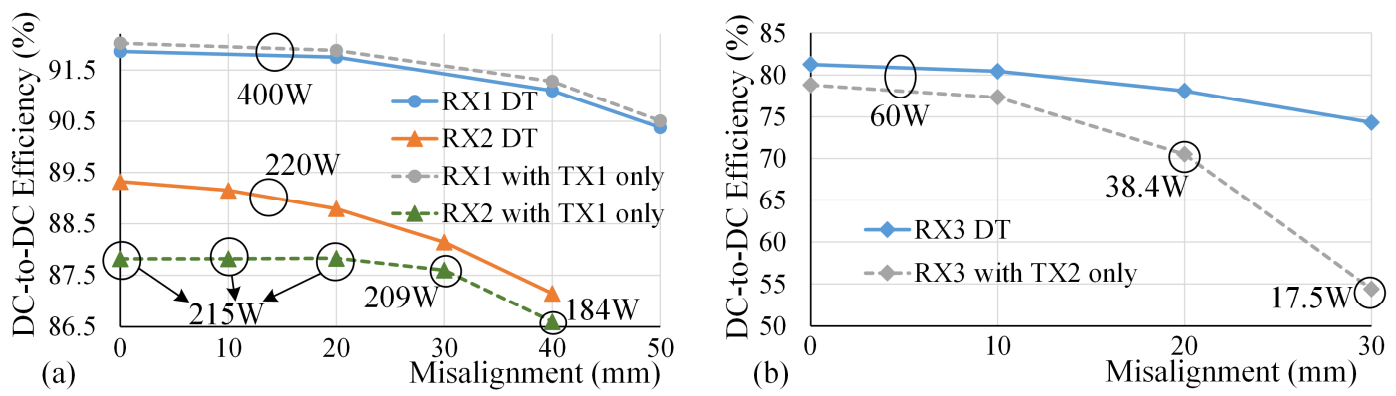

Fig. 7. Comparisons of efficiency and output power between dual-TX and single-TX systems. (a) RX1 and RX2 are paired with the TXs. (b) RX3 is paired with the TXs.

\section{Conclusion}

A dual-TX design concept is discussed for low-power inductive power transfer applications where compatibility with different-sized RXs and good misalignment tolerance are required. By energizing the magnetically decoupled LCC-compensated TXs using optimized voltages, the DC-to-DC efficiency can be maximized. Experimental results show that compared to using two individual TXs, the proposed scheme is generally more efficient and achieves a higher stability of output power under a lower bill-of-materials cost. Therefore, the compatibility with different-sized RXs is greatly improved. In future work, a DC/DC converter can be incorporated into the RX side and the well-established P\&O method can be applied in closed-loop feedback control. Besides, the system efficiency can be further improved by optimizing the circuit parameters to realize soft-switching of the inverters over a wider range of operating points.

\section{Acknowledgments}

This work was supported by Beijing Natural Science Foundation (No. 3212030).

\section{References}

[1] K. Fotopoulou and B. W. Flynn, Wireless Power Transfer in Loosely Coupled Links: Coil Misalignment Model, IEEE Transactions on Magnetics, vol. 47, no. 2, pp. 416-430, Feb. 2011.

[2] W. X. Zhong, X. Liu and S. Y. R. Hui, A Novel Single-Layer Winding Array and Receiver Coil Structure for Contactless Battery Charging Systems with Free-Positioning and Localized Charging Features, IEEE Transactions on Industrial Electronics, vol. 58, no. 9, pp. 4136-4144, Sept. 2011.

[3] S. Huh and D. Ahn, "Two-Transmitter Wireless Power Transfer with Optimal Activation and Current Selection of Transmitters," in IEEE Transactions on Power Electronics, vol. 33, no. 6, pp. 4957-4967, June 2018.

[4] D. Kim, S. Kim, S. Kim, J. Moon, I. Cho and D. Ahn, Coupling Extraction and Maximum Efficiency Tracking for Multiple Concurrent Transmitters in Dynamic Wireless Charging, IEEE Transactions on Power Electronics, vol. 35, no. 8, pp. 7853-7862, Aug. 2020.

[5] H. Feng, T. Cai, S. Duan, J. Zhao, X. Zhang and C. Chen, An LCC-Compensated Resonant Converter Optimized for Robust Reaction to Large Coupling Variation in Dynamic Wireless Power Transfer, IEEE Transactions on Industrial Electronics, vol. 63, no. 10, pp. 6591-6601, Oct. 2016.

[6] Y. Chen, B. Yang, Z. Kou, Z. He, G. Cao and R. Mai, Hybrid and Reconfigurable IPT Systems With HighMisalignment Tolerance for Constant-Current and Constant-Voltage Battery Charging, IEEE Transactions on Power Electronics, vol. 33, no. 10, pp. 8259-8269, Oct. 2018. 


\section{Authors}

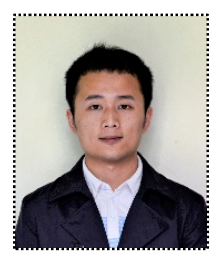

Guodong Zhu was born in Zhejiang, China, in 1995. He received the B.S. degree in Automotive Engineering from Tsinghua University, Beijing, China, in 2017, where he is currently working toward the Ph.D. degree in Power Engineering and Engineering Thermo-physics. His current research interest is wireless power transfer, particularly focusing on high-power electric vehicle wireless charging technology.

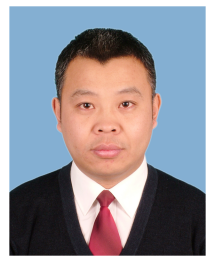

Dawei Gao was born in Jilin, China, in 1971. He received the B.S. degree from Southwest Jiaotong University, Sichuan, China, in 1992 and the Ph.D. degree from North China Electric Power University, Beijing, China, in 2001. From 2001 to 2003, he was a Postdoctoral Researcher with the Department of Automotive Engineering, Tsinghua University. From 2003 to 2005, he was an Assistant Researcher. Since 2005, he has been an Associate Researcher with the same department. His research interests include DC-DC converters, electric vehicle wireless power transfer, automotive power electronics and electric drive, and electric vehicle powertrain optimization. 